\title{
4.3 Problemstrukturen
}

Der zweite AEP-Erklärungsfaktor »Problemstrukturen« geht davon aus, dass jedes Politikfeld sich durch je eigene Problemstrukturen auszeichnet, die es von anderen Politikfeldern unterscheidet. Böcher/Töller (2012: 89) sprechen von der »Beschaffenheit der in einem Politikfeld behandelten Probleme«. Eine einflussreiche Referenz in diesem Diskurs ist Theodore Lowi, der bereits in den 1970er Jahren erkannte, dass eben diese Problemstrukturen die politischen Prozesse in einem Politikfeld maßgeblich prägen (Böcher/Töller 2012: 89; Döhler 2015: 57).

Was als »politisches Problem« gilt, ist indessen häufig umstritten - und selbst wenn eine Sachlage allgemein als politisches Problem anerkannt wird, befürworten verschiedene Akteure oftmals verschiedene Lösungswege (Reiter/Töller 2014: 101; Böcher/Töller 2012: 198). Insofern sind Problemstrukturen keinesfalls objektiv, sondern immer auch ein Ergebnis von Kämpfen über die Deutungshoheit und durchaus auch von Zufällen. Vielfach bringen etwa Katastrophen oder Skandale bereits lange bestehende Probleme erst auf die politische Tagesordnung (Zahariadis 2007: 69; Cairney/Zahariadis 2016: 90; vgl. Kapitel $4.4 \mathrm{zu}$ situativen Aspekten). Außerdem eignet sich nicht jedes Problem gleichermaßen für eine politische Inszenierung: Komplexe Sachlagen etwa sind nur schwer für eine breitere Öffentlichkeit aufzubereiten (Reiter/Töller 2014: 101; Böcher/Töller 2012: 193). Zudem ist das Risiko von politischen Fehlentscheidungen deutlich größer in einem von Unsicherheit geprägten Setting, weshalb sich mit Blick auf Wiederwahlinteressen auf leichter lösbare Probleme konzentriert werden könnte. Und schließlich beeinflusst auch die Frage der Betroffenheit, ob ein Problem als politisches Problem aufgegriffen wird: Würde die Problemlösung großen oder mächtigen Bevölkerungsgruppen schaden, ist eine Problemadressierung mit dem politischen Kalkül der Wiederwahl schwer zu vereinbaren (Böcher/Töller 2012: 193). Hier zeigen sich bereits Querverbindungen zum AEP-Erklärungsfaktor »Akteure und ihre Handlungen« (vgl. Kapitel 4.6). In diesem Kontext benennt Zahariadis (2007: 72) durch die begrenzte Aufnahme- und Verarbeitungskapazität politischer Entscheider*innen einen hohen "problem load«als hinderlich für die Entwicklung eines neuen politischen Problems. Dieses Merkmal bezieht sich darauf, wie stark die politische und mediale Öffentlichkeit ihre Aufmerksamkeit gerade anderen Themen widmet und entsprechend überhaupt Ressourcen für »neue« Probleme freisetzen kann.

Die bisherigen Ausführungen sind mit drei Problemtypen nach Roberts (2000: 1) zusammenzufassen: Bei einfachen Problemen besteht Konsens über die Problemdefinition und über die Problemlösung, sodass derartige Probleme politisch leicht adressierbar sind. Komplexe Probleme zeichnen sich hingegen dadurch aus, dass die beteiligten Akteure sich zwar in der Problemdefinition einig sind, der Weg zur Lösung dieses Problems jedoch umstritten ist. Und bei sogenannten »wicked problems« - wahlweise mit »heimtückisch«, »gefährlich« oder »bösartig« übersetzt, aber meist beim englischen Terminus verbleibend (vgl. Kemmerzell 2017: 246) - besteht weder ein Konsens darüber, was eigentlich das Problem ist, noch darüber, wie dieses nicht einheitlich definierte Problem politisch adressiert werden soll (Mumm 2016: 55ff.; Zohlnhöfer/Rüb 2016: 57f.; Bandelow/Hegelich 2011: 20). Die Konflikthaftigkeit im politischen Prozess nimmt mit 
steigender Komplexität der Probleme $\mathrm{zu}$ und ist folglich bei »wicked problems « am größten (Roberts 2000: 1, vgl. auch Böcher/Töller 2019: 105f.).

Im Kontext des globalen Klimawandels haben Levin et al. (2012: 124) noch die »super wicked problems« entwickelt, die generell die Problemcharakteristika der »wicked problems « teilen, aber erschwerend noch andere Problemstrukturen enthalten. Erstens handelt es sich bei solchen Problemen um besonders drängende, da bei politischem Nichthandeln »die Zeit knapp wird«. Zweitens sind die Problemverursacher* innen gleichzeitig diejenigen, die für die Lösung des Problems zuständig wären. Drittens gibt es nur eine schwache oder gar keine zentrale Entscheidungsebene und viertens führen die vorhergenannten Punkte dazu, dass trotz der knapper werdenden Zeit, die zur Problemlösung verbleibt, ein Aufschieben in die Zukunft wahrscheinlich ist.

Welchem Problemtyp der barrierefreie bzw. -reduzierte Wohnraum zuzuordnen ist, wird im Laufe der empirischen Analysen zu überprüfen sein. Wie oben gezeigt wurde, ist die Interpretation einer Problemstruktur jedoch nie rein objektiv und hängt von der Betroffenheit der jeweiligen Akteure ab (vgl. Reiter/Töller 2014: 101; Böcher/Töller 2012: 198).

\subsection{Situative Aspekte}

Die Diskussion der Instrumenten-Alternativen (vgl. Kapitel 4.2.2) hat bereits auf den inkrementellen Politikstil und die damit verbundene Relevanz von zeitlichen Abfolgen hingewiesen, die einer bestimmten Maßnahme zur Durchsetzung verhelfen können. Diesem Umstand widmet sich der AEP-Erklärungsfaktor »situative Aspekte« dezidiert. So geht der AEP nicht davon aus, dass politische Probleme im Sinne eines linearen Problemlösungsprozesses von politischen Akteuren aufgegriffen werden und diese daraufhin nach der rationalsten Lösung suchen. Vielmehr leiten gesellschaftliche Stimmungen sowie Interessen das politische Geschehen. Dies kann relativ unabhängig von dem sachgegebenen Handlungsdruck eines bestimmten Problems erfolgen (Böcher/Töller 2012: 190f.; Cairney/Zahariadis 2016: 87; vgl. auch Kapitel 4.3 zu Problemstrukturen).

Die Idee situativer Aspekte wurde bereits in den 1980er Jahren im MSA integriert: Derartige »focusing events « eröffnen ein sogenanntes Möglichkeitsfenster für politische Veränderungen (Zahariadis 2016: 12). Es ist schließlich bereits aus der empirischen Beobachtung heraus evident, dass aktuelle Ereignisse die politische Stimmung beeinflussen und in Ausnahmefällen sogar umkehren können. Ein prominentes Beispiel sind Umweltkatastrophen wie etwa das Waldsterben der 1980er Jahre oder die nuklearen Unfälle in Tschernobyl (1986) und Fukushima (2011). So beschloss die damalige schwarzgelbe Regierung in Reaktion auf das Unglück in Fukushima einen überraschend entschiedenen Ausstieg aus der Kernenergie (Reiter/Töller 2014: 103, vertiefend Bernardi et al. 2018). Aktueller kann die Corona-Pandemie als situativer Aspekt gelten, weil diese nicht nur politische Aufmerksamkeit bündelt, sondern zur Überwindung der durch die Pandemie ausgelösten wirtschaftlichen Krise auch erhebliche finanzielle Mittel gebunden werden. Angesichts des oben erläuterten »problem loads« steht zu erwarten, dass andere politische Probleme zumindest mittelfristig in den Hintergrund treten werden. 